(c) American Dairy Science Association, 2005.

\title{
Hot Topic: Endogenous Production of $n-3$ and n-6 Fatty Acids in Mammalian Cells
}

\author{
K. C. Morimoto, ${ }^{\star}$ A. L. Van Eenennaam, ${ }^{*}$ E. J. DePeters, and J. F. Medrano \\ Department of Animal Science, University of California, Davis 95616
}

\section{ABSTRACT}

Polyunsaturated fatty acids (PUFA) are important components of mammalian diets, and the beneficial effects of n-3 PUFA on human development and cardiovascular health have been well documented. Caenorhabditis elegans is one of the few animals known to be able to produce linoleic (LA, 18:2n-6) and $\alpha$-linolenic (ALA, 18:3n-3) essential fatty acids. These essential PUFA are generated by the action of desaturases that successively direct the conversion of monounsaturated fatty acids (MUFA) to PUFA. The cDNA coding sequences of the C. elegans $\Delta^{12}$ and n-3 fatty acid desaturases were each placed under the control of separate constitutive eukaryotic promoters and simultaneously introduced into HC11 mouse mammary epithelial cells by adenoviral transduction. Phospholipids from transduced cells showed a significant decrease in the ratios of both MUFA:PUFA and n-6:n-3 fatty acids relative to control cultures. The fatty acid profile of transduced cellular phospholipids revealed significant decreases in MUFA and arachidonic acid (20:4n-6), and increases in LA, ALA, and eicosapentaenoic acid (20:5n-3). The fatty acid composition of triacylglycerols derived from transduced cells was similarly, but less dramatically, affected. These results demonstrate the functionality of C. elegans fatty acid desaturase enzymes in mammalian cells. Expression of these desaturases in livestock might act to counterbalance the saturating effect that rumen microbial biohydrogenation has on the fatty acid profile of ruminant products, and allow for the development of novel, land-based dietary sources of n-3 PUFA. (Key words: polyunsaturated fatty acid, n-3, fatty acid desaturase, transgenic)

Abbreviation key: ALA $=\alpha$-linolenic acid, $\mathbf{C o A}=$ coenzyme A, FAT-1 = n-3 fatty acid desaturase, FAT-2 = $\Delta^{12}$ fatty acid desaturase, LA = linoleic acid, MUFA =

Received December 8, 2004.

Accepted January 6, 2005.

Corresponding author: A. L. Van Eenennaam; e-mail: alvaneenennaam@ucdavis.edu.

*These 2 authors contributed equally to this work. monounsaturated fatty acids, PUFA = polyunsaturated fatty acids, SFA = saturated fatty acids .

Long-chain polyunsaturated fatty acids (PUFA) play a particularly important role in fetal development and the maintenance of overall human health, either as a component of membrane phospholipids, or as precursors to various eicosanoids (Jump, 2002). The most abundant PUFA are often described as n-3 (omega-3) or n-6 (omega-6), which refers to the number of the first carbon with a double bond relative to the carbon at the methyl end of the molecule. Because n-3 and n-6 PUFA are not interconvertible in mammals, elevating tissue concentrations of n-3 PUFA relies on chronic dietary intake of fats rich in n-3 PUFA. It has been observed that the ratio of $n-6: n-3$ PUFA present in the diet of industrial societies has increased as a result of the greater consumption of vegetable oils rich in n-6 fatty acids and a reduced consumption of fish and plant sources of n-3 fatty acids. This imbalance has been linked to an increased risk of coronary heart disease and other human ailments (Connor, 2000; Simopoulos, 2004). According to current dietary customs, the consumption of fish is the most practical source of long chain n-3 PUFA and concern exists as to whether the current fish-based supply is adequate to meet future needs (Pauly et al., 2002). To supply the requirements of a growing population, long-chain n-3 PUFA sources that are renewable and sustainable need to be developed.

Animal products represent a large proportion of the Western diet and are an important source of protein. Cattle contribute $36.1 \%$ of all protein consumed, whereas fish supply only $5.5 \%$ of total protein (Smit et al., 1999). Beef and dairy products contain low levels of PUFA because of the extensive fatty acid biohydrogenation carried out by the rumen microbial population. Increasing the proportion of PUFA in ruminant products could have significant human health benefits (Visioli et al., 2000). One approach to achieve this goal has been to treat dietary feedstuffs with heat or chemicals to protect the PUFA from rumen biohydrogenation (Noakes et al., 1996). This approach has met with only limited success and remains dependent upon the provi- 


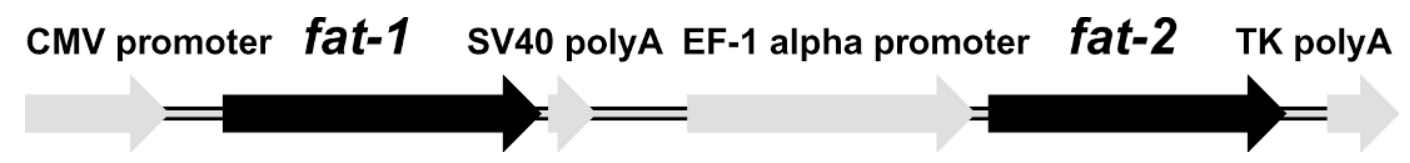

Figure 1. Schematic representation of the 5475-bp expression construct containing the Caenorhabditis elegans n-3 (fat-1; GenBank accession number L41807) and $\Delta^{12}$ (fat-2; GenBank accession number NM_070159) fatty acid desaturases under the control of constitutive eukaryotic promoters.

sion of exogenous PUFA in the feedstuff. An alternative approach to develop novel land-based sources of PUFA would be to genetically engineer animals to produce their own desaturase enzymes to allow for de novo fatty acid desaturation.

Vertebrates possess the stearoyl coenzyme A (CoA)desaturase enzyme required to synthesize monounsaturated fatty acids (MUFA) from saturated fatty acids (SFA). However, they lack the fatty acid desaturase enzymes required for the synthesis of linoleic acid (LA, 18:2n-6) and $\alpha$-linolenic acid (ALA, 18:3n-3), and are therefore dependent on dietary sources of these essential PUFA. The nematode Caenorhabditis elegans is able to synthesize both LA and ALA by virtue of an endogenous n-3 fatty acid desaturase (FAT-1) that recognizes a range of 18- and 20-carbon $\mathrm{n}-6$ substrates (Spychalla et al., 1997) and a $\Delta^{12}$ fatty acid desaturase (FAT-2) that converts 16- and 18-carbon MUFA to n6 fatty acids (Peyou-Ndi et al., 2000). We hypothesized that simultaneous expression of FAT-1 and FAT-2 in mammalian cells would enable the conversion of MUFA to n-3 PUFA and thus, the endogenous production of both LA and ALA essential fatty acids.

To test this hypothesis, FAT-1 and FAT-2 were constitutively expressed in $\mathrm{HC} 11$ mouse mammary epithelial cells. HC11 is a line of cells originally derived from COMMA-1D, an immortal cell line established from the mammary tissue of midpregnant BALB/c mice. This cell line can be grown using simplified cell culture conditions because it has no requirements for the addition of an exogenous extracellular matrix or co-cultivation with other cell types (Ball et al., 1988). Cells were maintained in growth medium consisting of RPMI+L-Glutamine (Gibco, Carlsbad, CA), supplemented with $8 \%$ heat-inactivated fetal calf serum (Gibco), $10 \mathrm{ng} / \mathrm{mL}$ epidermal growth factor (Sigma Chemical Co., St. Louis, MO), $5 \mu \mathrm{g} / \mathrm{mL}$ bovine insulin (Sigma Chemical Co.), and $50 \mu \mathrm{g} / \mathrm{mL}$ gentamicin (Gibco) in a humidified incubator at $37^{\circ} \mathrm{C}+5 \% \mathrm{CO}_{2}$ (Marte et al., 1994). Cells were subcultured at a ratio of 1:5 every $3 \mathrm{~d}$. HC11 cells were plated at $2 \times 10^{6}$ cells per $60-\mathrm{mm}$ Petri dish on the day before infection with the prepared adenovirus.

To construct the adenoviral expression vector, the $C$. elegans fat-1 (GenBank accession number L41807) and fat-2 (GenBank accession number NM_070159) cDNA sequences were placed under the control of the CMV and EF-1 alpha constitutive promoters in pBudCE4.1 (Invitrogen, Carlsbad, CA) respectively (Figure 1), and subcloned into the Gateway pENTR 2B Vector (Invitrogen). A recombination reaction between the $a t t \mathrm{~L}$ and att $\mathrm{R}$ attachment sites was performed to insert the desaturase genes into the $\mathrm{pAd} / \mathrm{CMV} / \mathrm{V} 5 / \mathrm{DEST}$ Gateway Vector (Invitrogen) containing the human adenoviral gene, resulting in the $\mathrm{pAd} /$ fat-1/fat-2 construct.

The 293A cell line of human embryonic kidney cells expressing the human adenovirus E1 proteins (Invitrogen) was used to produce the recombinant adenovirus. The cells were maintained in complete growth media consisting of DMEM (high glucose) (Gibco), 10\% heat inactivated fetal bovine serum (Gibco), $0.1 \mathrm{mM}$ MEM Nonessential Amino Acids (Gibco), $2 \mathrm{~m} M$ L-glutamine (Gibco), and $1 \%$ penicillin-streptomycin (Gibco) in a humidified incubator at $37^{\circ} \mathrm{C}+5 \% \mathrm{CO}_{2}$. Cells were subcultured at $\sim 75$ to $85 \%$ confluence and fed complete growth media every $3 \mathrm{~d}$. Cell density and viability were determined with a hemocytometer chamber and trypan blue exclusion (Graham et al., 1977).

To produce the adenovirus, both $\mathrm{pAd} /$ fat-1/fat-2 and a control plasmid ( $\mathrm{pAd} / \mathrm{CMV} / \mathrm{V} 5-\mathrm{GW} / \mathrm{lacZ}$, Invitrogen) were digested with $P a c$ I to expose left and right viral inverted terminal repeats. Fragments were purified with a QIAprep Spin Miniprep Kit (Qiagen, Valencia, CA). The 293A cells were plated in 6 -well plates at $5 \times$ $10^{5}$ cells/well. The following day, at approximately $90 \%$ confluency, the cultures were transfected with $\mathrm{pAd} /$ fat1/fat-2 or pAd/CMV/V5-GW/lacZ using Lipofectamine 2000 (Invitrogen) according to the manufacturer's protocol with $3 \mu \mathrm{L}$ of Lipofectamine 2000 and $1 \mu \mathrm{g}$ of DNA per well. At $48 \mathrm{~h}$ post-transduction, the cells were transferred to a T-75 flask with complete growth media. Media were replaced every $3 \mathrm{~d}$ until cytopathic effects were observed, which was at approximately $12 \mathrm{~d}$ post-transduction. Approximately $18 \mathrm{~d}$ post-transduction, cytopathic effects had spread to $80 \%$ of the culture and plaques were visible. The cells and culture media were removed from the flasks and subjected to 3 rounds of freeze/thaw at $-80^{\circ} \mathrm{C}(30 \mathrm{~min})$ and $37^{\circ} \mathrm{C}(15 \mathrm{~min})$. The cell lysate was centrifuged, and the supernatant containing the $\mathrm{Ad} /$ fat-1/fat-1 or $\mathrm{Ad} / \mathrm{lacZ}$ viral lysate was aliquoted, stored at $-80^{\circ} \mathrm{C}$, and titered before use. 
HC11 cells were infected with either the Ad/fat-1/fat2 or the $A d / l a c Z$ virus at a multiplicity of infection of 10 . One culture transduced with $A d / l a c Z$ virus was stained $3 \mathrm{~d}$ post-transduction using a $\beta$-galactosidase staining kit (Mirus, Madison, WI) to determine the efficiency of transduction (Figure 2). The adenoviral system resulted in a very high efficiency $(\sim 95 \%)$ of transduction and was found to be much more effective as a means of gene delivery to HC11 cells than lipofection (data not shown).

Three days post-transduction, the infected $\mathrm{HC} 11$ cells were rinsed with PBS (Gibco) and homogenized. Expression of FAT-1 protein in cultures transduced with $\mathrm{Ad} /$ fat-1/fat-2 virus was confirmed by Western analysis (data not shown). Lipids were extracted in chloroformmethanol $(2: 1, \mathrm{vol} / \mathrm{vol})$ containing $0.2 \%$ glacial acetic acid and dried under nitrogen at $60^{\circ} \mathrm{C}$; the lipids were resuspended in hexane. Separation of polar and nonpolar lipids was performed with a Sep-pak silica cartridge WAT43400 (Waters, Milford, MA). Nonpolar lipids were eluted with hexane:ethyl ether (1:1, vol/vol). Polar lipids were eluted with methanol and chloroform:methanol:water (3:5:2, vol:vol:vol). Both samples were evaporated under nitrogen at $60^{\circ} \mathrm{C}$ and resuspended in $2 \mathrm{~mL}$ of iso-octane. The fatty acid profiles of the lipid fractions were analyzed via gas chromatography (DePeters et al., 2001).

Statistical analysis of cellular fatty acid profiles from 6 experimental replicates per virus treatment $(\mathrm{Ad} /$ fat 1/fat-2 or the $\mathrm{Ad} / l a c Z$ virus) was performed using a 1way ANOVA. To examine the effect of the desaturase transgenes on the ratios of MUFA (16:1 + 18:1):PUFA $(18: 2+18: 3+20: 4+20: 5)$ and $n-6(18: 2+20: 4): n-3$ $(18: 3+20: 5)$ fatty acids, the variance and covariance of the ratios were used to calculate the standard error of the difference between ratios (Cochran, 1977). A onetail test of the hypothesis of the difference between the 2 ratios was performed using the calculated standard error: $R-R^{\prime} \pm 1.65 \times S E\left(R-R^{\prime}\right)$ where $R$ and $R^{\prime}$ represent the ratios being compared, $\mathrm{SE}\left(\mathrm{R}-\mathrm{R}^{\prime}\right)$ the standard error of the difference between the 2 ratios, and $1.65=z_{0.05}$.

Phospholipids from mammalian cells expressing FAT-1 and FAT-2 showed a significant $(P<0.05)$ decrease in the ratios of both MUFA:PUFA (1.08 vs.1.81) and $n-6: n-3$ fatty acids (0.81 vs. 6.81$)$ relative to the ratios found in control cultures expressing lacZ, respectively. The fatty acid profile of cells expressing FAT-1 and FAT-2 revealed an increase in LA, reflecting the known $\Delta^{12}$-desaturase activity of FAT-2; and both a decrease in arachidonic acid (20:4n-6) and increases in ALA and eicosapentaenoic acid (20:5n-3), reflecting the known n-3 desaturase activities of FAT-1 (Figure 3a). Although LA is a substrate for FAT-1, the decrease in
LA due to desaturation by FAT-1 was likely overshadowed by the influx of LA from the desaturation of oleic acid by FAT-2. The fatty acid composition of triacylglycerols derived from transfected cells was similarly, but less dramatically, affected (Figure 3b).

Because mammals lack the desaturase enzymes necessary to synthesize LA and ALA, they are dependent upon dietary sources of these essential fatty acids. In this experiment, LA and ALA constituted a relatively small proportion $(2.45 \%)$ of phospholipid fatty acids in cultures that were transduced with $\mathrm{Ad} / l a c Z$ virus. However, in the cultures that were transduced with $\mathrm{Ad} /$ fat1/fat-2 virus, the combined proportion of LA and ALA in the phospholipid fatty acid pool was significantly higher $(6.45 \%)$. These results suggest that the cultures that were expressing FAT-1 and FAT-2 were successfully synthesizing essential fatty acids endogenously, utilizing a fatty acid desaturation pathway that is normally absent from mammalian cells.

Unlike mammalian desaturases that act on fatty acyl-CoA triacylglycerol precursors (Pereira et al., 2003), FAT-1 and FAT-2 are hypothesized to be acyllipid desaturases. This may explain why the desaturases appeared to have a more immediate effect on the composition of phospholipid-bound acyl groups. This speculation is supported by the finding that the FAT-1 enzyme was found to desaturate the 18:2 of Arabidopsis membrane lipids (Spychalla et al., 1997), and by the fact that the LA product of a fungal $\Delta^{12}$ acyl-lipid fatty acid desaturase expressed in mammalian cells was exclusively located in the cellular phospholipid fraction (Kelder et al., 2001).

The principal route of triacylglyceride biosynthesis involves the transfer of acyl-CoA to a glycerol backbone via the Kennedy pathway (Kennedy, 1961), although triacylglycerols can also be synthesized from the products of phospholipid hydrolysis. Transgenic mice constitutively expressing fat-1 were found to have a significant decrease in the n-6:n-3 fatty acid ratio of milk fat (Kang et al., 2004), suggesting the movement of $n-3$ fatty acids from phospholipids into the triacylglycerol pool ( $98 \%$ of milk fat). Interestingly, there were no apparent health problems in these transgenic mice although some may have been anticipated given the importance of phospholipid membrane composition on cellular metabolism (Hulbert, 2003). Imbalances in PUFA are attributed to many chronic disease states (Sanders, 1993), and can influence prostaglandin synthesis and fertility (Abayasekara and Wathes, 1999; Robinson et al., 2002). In vivo experiments will be required to establish what impact the coexpression of FAT-1 and FAT2 desaturase enzymes might have on animal health and the PUFA content of milk and meat products derived from ruminant livestock. 


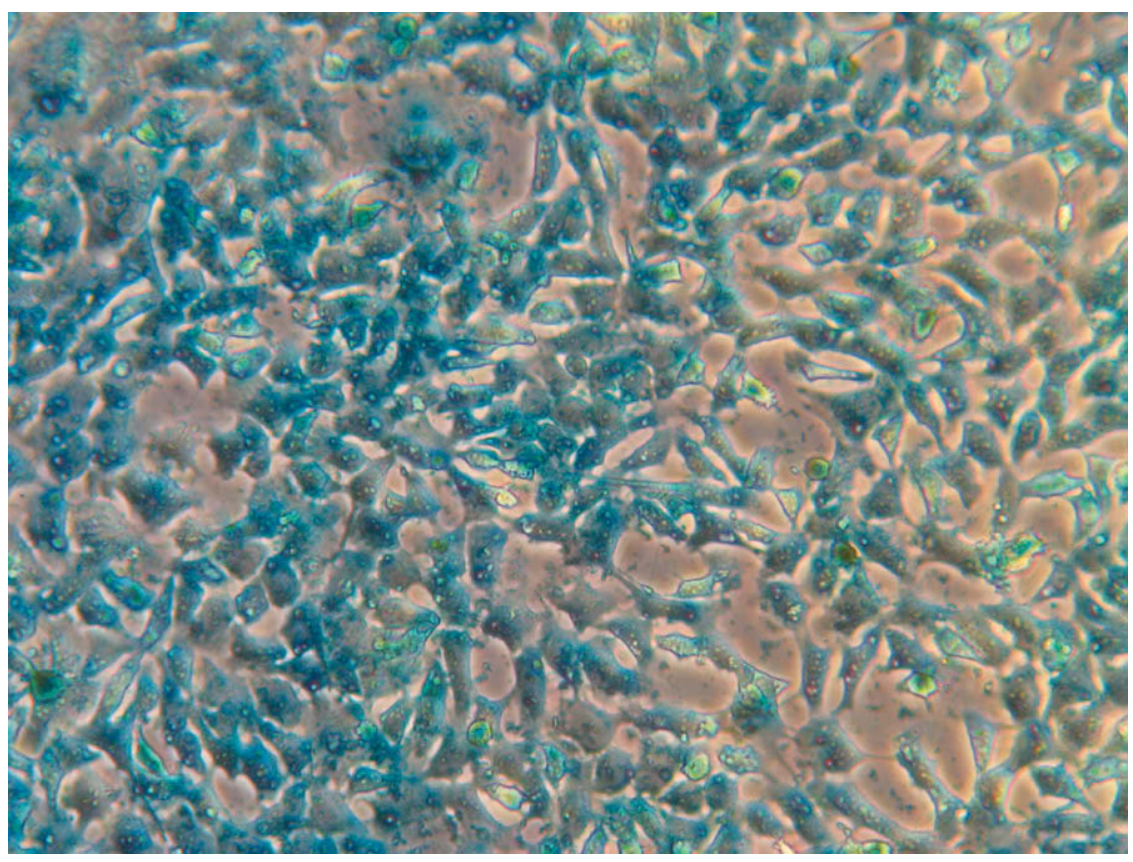

Figure 2. HC11 cells 3 d post-transduction with Ad/LacZ stained with $\beta$-galactosidase. Blue cells indicate successfully transfected cells showing a transduction efficiency of about $95 \%$ (20× magnification).

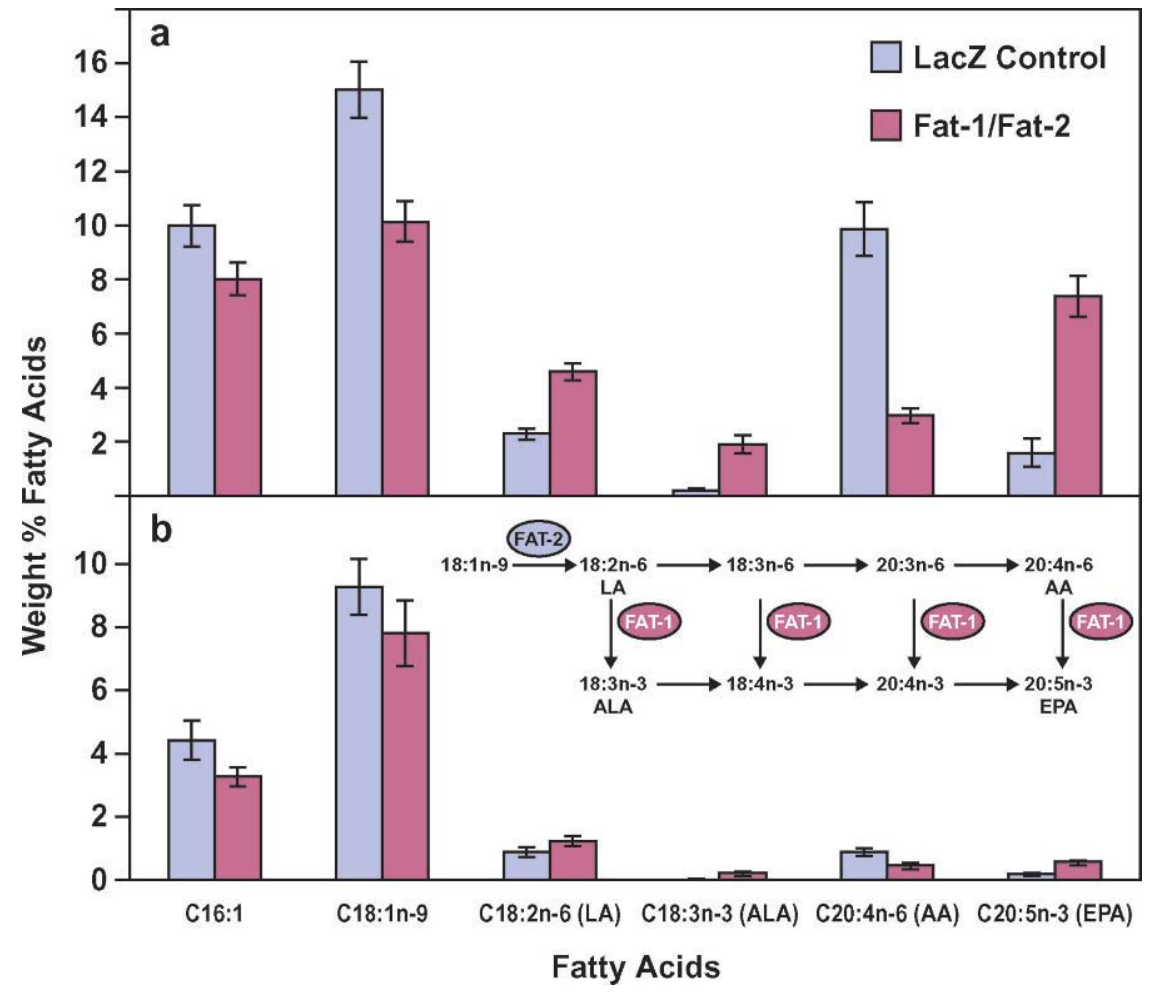

Figure 3. Fatty acid profile of cellular lipids as determined by gas chromatography. Fatty acid composition of the (a) phospholipid, and (b) triacylglycerol fraction of HC11 mouse mammary epithelial cells assayed $3 \mathrm{~d}$ after adenoviral-mediated transduction with recombinant virus containing Caenorhabditis elegans $\Delta^{12}$ (FAT-2) and n-3 (FAT-1) fatty acid desaturases or LacZ under the control of constitutive promoters. Error bars represent SEM $(\mathrm{n}=6)$. Inset: FAT-1 and FAT-2 mediated fatty acid desaturation pathways. LA = linoleic acid, ALA = alpha-linolenic acid, AA = arachidonic acid, EPA = eicosapentaenoic acid. 
This study demonstrates that the expression of $C$. elegans fatty acid desaturase genes in mammalian cells can significantly alter the cellular fatty acid profile and suggests a transgenic approach to counteract the rumen microbial biohydrogenation of unsaturated fatty acids by enabling the endogenous production of PUFA. Increasing the n-3 PUFA of beef and dairy products offers a way to improve the nutritional content of an important component of the Western diet within the realm of existing food preferences, and would provide a compelling example of how biotechnology could be employed to produce functional foods for the enhancement of human health.

\section{ACKNOWLEDGMENTS}

We thank Scott Taylor for technical assistance with fatty acid analyses, and John Browse, Institute of Biological Chemistry, Washington State University (Pullman, WA) for generously providing $C$. elegans fat-1 and fat-2 cDNA. This study was supported by NIH grant 1R03HD047193-01 (to A.L.V.) and the UC Davis Dairy Milk Components Laboratory.

\section{REFERENCES}

Abayasekara, D. R., and D. C. Wathes. 1999. Effects of altering dietary fatty acid composition on prostaglandin synthesis and fertility. Prostaglandins Leukot. Essent. Fatty Acids 61:275-287.

Ball, R. K., R. R. Friis, C. A. Schoenenberger, W. Doppler, and B. Groner. 1988. Prolactin regulation of beta-casein gene expression and of a cytosolic 120-kd protein in a cloned mouse mammary epithelial cell line. EMBO J. 7:2089-2095.

Cochran, W. 1977. Sampling Techniques. 3rd ed. Wiley, New York, NY.

Connor, W. E. 2000. Importance of n-3 fatty acids in health and disease. Am. J. Clin. Nutr. 71(1 Suppl.):171S-175S.

DePeters, E., J. German, S. Taylor, S. Essex, and H. PerezMonti. 2001. Fatty acid and triglyceride composition of milk fat from lactating Holstein cows in response to supplemental canola oil. J. Dairy Sci. 84:929-936.

Graham, F. L., J. Smiley, W. C. Russell, and R. Nairn. 1977. Characteristics of a human cell line transformed by DNA from human adenovirus type 5. J. Gen. Virol. 36:59-74.
Hulbert, A. J. 2003. Life, death and membrane bilayers. J. Exp. Biol. 206:2303-2311.

Jump, D. B. 2002. The biochemistry of n-3 polyunsaturated fatty acids. J. Biol. Chem. 277:8755-8758.

Kang, J. X., J. Wang, L. Wu, and Z. B. Kang. 2004. Transgenic mice: fat-1 mice convert n-6 to n-3 fatty acids. Nature 427:504.

Kelder, B., P. Mukeji, S. Kirchner, G. Hovanec, A. E. Leonard, L. T. Chuang, J. J. Kopchick, and Y. S. Huang. 2001. Expression of fungal desaturase genes in cultured mammalian cells. Mol. Cell. Biochem. 219:7-11.

Kennedy, E. P. 1961. Biosynthesis of complex lipids. Fed. Proc. 20:934-940.

Marte, B. M., T. Meyer, S. Stabel, G. J. Standke, S. Jaken, D. Fabbro, and N. E. Hynes. 1994. Protein kinase C and mammary cell differentiation: Involvement of protein kinase $\mathrm{C}$ alpha in the induction of beta-casein expression. Cell. Growth Differ. 5:239-247.

Noakes, M., P. J. Nestel, and P. M. Clifton. 1996. Modifying the fatty acid profile of dairy products through feedlot technology lowers plasma cholesterol of humans consuming the products. Am. J. Clin. Nutr. 63:42-46.

Pauly, D., V. Christensen, S. Guenette, T. J. Pitcher, U. R. Sumaila, C. J. Walters, R. Watson, and D. Zeller. 2002. Towards sustainability in world fisheries. Nature 418:689-695.

Pereira, S. L., A. E. Leonard, and P. Mukerji. 2003. Recent advances in the study of fatty acid desaturases from animals and lower eukaryotes. Prostaglandins Leukot. Essent. Fatty Acids 68:97106.

Peyou-Ndi, M. M., J. L. Watts, and J. Browse. 2000. Identification and characterization of an animal delta(12) fatty acid desaturase gene by heterologous expression in Saccharomyces cerevisiae. Arch. Biochem. Biophys. 376:399-408.

Robinson, R. S., P. G. Pushpakumara, Z. Cheng, A. R. Peters, D. R. Abayasekara, and D. C. Wathes. 2002. Effects of dietary polyunsaturated fatty acids on ovarian and uterine function in lactating dairy cows. Reproduction 124:119-131.

Sanders, T. A. 1993. Marine oils: Metabolic effects and role in human nutrition. Proc. Nutr. Soc. 52:457-472.

Simopoulos, A. P. 2004. Omega-6/Omega-3 Essential Fatty Acid Ratio and Chronic Diseases. Vol. 20. Marcel Dekker, New York, NY.

Smit, E., F. J. Nieto, C. J. Crespo, and P. Mitchell. 1999. Estimates of animal and plant protein intake in US adults: Results from the Third National Health and Nutrition Examination Survey, 1988-1991. J. Am. Diet. Assoc. 99:813-820.

Spychalla, J. P., A. J. Kinney, and J. Browse. 1997. Identification of an animal omega-3 fatty acid desaturase by heterologous expression in Arabidopsis. Proc. Natl. Acad. Sci. USA. 94:1142-1147.

Visioli, F., P. Rise, E. Plasmati, F. Pazzucconi, C. R. Sirtori, and C. Galli. 2000. Very low intakes of n-3 fatty acids incorporated into bovine milk reduce plasma triacylglycerol and increase HDLcholesterol concentrations in healthy subjects. Pharmacol. Res. 41:571-576. 